\title{
Pulmonary antioxidant concentrations and oxidative damage in ventilated premature babies
}

\author{
K J Collard, S Godeck, J E Holley, M W Quinn
}

Arch Dis Child Fetal Neonatal Ed 2004;89:F412-F416. doi: 10.1136/adc.2002.016717

See end of article for authors' affiliations

Correspondence to

Dr Collard, Department of Medical Sciences, St Loye's School of Health Studies, Millbrook House,

Millbrook Lane, Topsham Road, Exeter EX2 6ES, UK; k.j.collard@exeter.ac.uk

Accepted 2 July 2003
Objective: To determine the relation between lipid peroxidation and the antioxidants ascorbate, urate, and glutathione in epithelial lining fluid in ventilated premature babies, and to relate the biochemical findings to clinical outcome.

Design: A cohort study conducted between January 1999 and June 2001.

Setting: A NHS neonatal intensive care unit.

Patients: An opportunity sample of 43 ventilated babies of less than 32 weeks gestation.

Main outcome measures: The duration of supplementary oxygen according to the definition of bronchopulmonary dysplasia (BPD; oxygen dependency at 36 weeks gestational age).

Methods: Epithelial lining fluid was sampled by bronchoalveolar lavage. Ascorbate, urate, glutathione, and malondialdehyde (a marker of lipid peroxidation) were measured.

Results: Babies who developed BPD had significantly lower initial glutathione concentrations (mean (SEM) $1.89(0.62) \vee 10.76(2.79) \mu \mathrm{M} ; \mathrm{p}=0.043$ ) and higher malondialdehyde concentrations (mean (SEM) $1.3(0.31) \vee 0.345(0.09) \mu M ; p<0.05)$ in the epithelial lining fluid than those who were not oxygen dependent. These variables were poor predictors of the development of BPD. Gestational age, endotracheal infection, and septicaemia had good predictive power. The level of oxidative damage was associated with the presence of endotracheal infection/septicaemia rather than inspired oxygen concentration.

Conclusions: Endotracheal infection, septicaemia, and gestational age, rather than antioxidant concentrations, are the most powerful predictors of the development of BPD.
$\mathrm{V}$ entilated premature babies are at risk of developing chronic lung disease of prematurity, bronchopulmonary dysplasia (BPD). Babies who develop BPD go on to have a higher rate of respiratory problems in later life and also have a higher incidence of neurodevelopmental disorders. ${ }^{1-3}$ BPD is a serious consequence of prematurity, and interventions aimed at limiting its occurrence would be valuable additions to the clinical care of these babies. To achieve this, a greater understanding of the causes of BPD is needed. Although a multifactorial condition, it is widely believed that BPD is due, at least in part, to oxidant free radical injury to the immature lung. ${ }^{4}$ The free radicals are generated as a consequence of the high levels of inspired oxygen and by respiratory bursts in invading inflammatory cells. ${ }^{5-7}$ Previous studies have provided biochemical evidence of pulmonary free radical damage in premature babies who develop chronic lung disease. ${ }^{89}$ Antioxidants present in the alveolar epithelial lining fluid (ELF) are well positioned to defend against tissue damage caused by free radicals generated by these mechanisms. ${ }^{10}$ These antioxidants can be measured in bronchoalveolar lavage (BAL) fluid. Among the non-enzyme antioxidants present in the alveolar spaces, there is evidence that ascorbate, urate, and glutathione are important. ${ }^{71-13}$ These antioxidants work together in a coordinated manner to defend against free radical damage. Glutathione is an important substrate in the detoxification of peroxide by glutathione peroxidase. ${ }^{14}$ It also plays an important role in promoting the reduced form of other antioxidants. ${ }^{15}$ The importance of glutathione in protecting the lung against oxidative damage has been well established in studies in animals in which hyperoxia or inhibition of glutathione synthesis led to significant oxidative damage to the lung, which could be ameliorated by treatment with glutathione esters or precursors. ${ }^{16}{ }^{17}$ In addition, babies who develop chronic lung disease have decreased concentrations of glutathione in BAL fluid. ${ }^{18}{ }^{19}$ Urate is a particularly important antioxidant because, in addition to acting as a free radical scavenger, ${ }^{11}$ it is able to chelate non-protein bound iron and to subsequently reduce iron induced oxidant activity, ${ }^{20}$ including iron induced oxidation of ascorbate. ${ }^{20}$ There is some evidence that urate may be important in protecting against oxidative damage in early neonatal life. ${ }^{12}$ Ascorbate is an important pulmonary antioxidant ${ }^{13}$ and also functions to recycle oxidised glutathione to its reduced form. ${ }^{15}$ Although the redox state of the antioxidants is important, the absolute availability is also relevant because, irrespective of redox state, a severe deficiency in any antioxidant could compromise the ability of the lung to regulate its overall antioxidant status and to defend against oxidative damage.

To understand the relation between antioxidant status, oxidative damage, and clinical outcome, a biochemical marker of oxidative damage that can also be measured in lung fluid is required. Lipid peroxidation is a likely form of oxidative damage in the developing lung. Malondialdehyde is one of the most abundant products of lipid peroxidation and has been shown to be a sensitive marker of oxidative damage in a number of tissues. ${ }^{22}{ }^{23}$ It has been identified in the urine of premature babies and shown to be increased in babies who develop chronic lung disease. ${ }^{24}$

The concentrations of malondialdehyde and glutathione, ascorbate, and urate were related to clinical outcome and to other potentially confounding variables. The aim was to test the hypothesis that there is an inverse relation between pulmonary oxidative damage and ELF antioxidant concentrations and that poor clinical outcome is associated with

Abbreviations: BAL, bronchoalveolar lavage; BPD, bronchopulmonary dysplasia; ELF, epithelial lining fluid 
high levels of oxidative damage and/or low antioxidant concentrations.

\section{PATIENTS AND METHODS Sample group}

The sample group consisted of ventilated babies of less than 32 weeks gestation for whom informed parental consent had been obtained and who were being cared for in the neonatal intensive care unit at the Royal Devon \& Exeter Hospital (Heavitree). The study was conducted between January 1999 and June 2001. Wherever possible, parents were fully informed of the study prenatally and "provisional consent" often obtained. ${ }^{25}$ Written consent was obtained in the early postnatal period in all cases.

During the study, a total of 117 babies of less than 32 weeks gestation were ventilated in our unit. The study recruited 45 babies, of whom one died before any samples could be taken and one provided insufficient samples for analysis, resulting in 43 babies for analysis.

\section{Clinical outcome}

Oxygen dependency was defined according to continuous saturation monitoring and an overnight trace at 36 weeks post-conceptional age, whether or not the baby had an oxygen requirement. If oxygen saturation was consistently below $92 \%$ in room air, the baby was classified as oxygen dependent. The 43 babies were divided into four groups according to clinical outcome in relation to oxygen dependency. Group 1 consisted of 17 babies who did not require supplementary oxygen at either 28 days postnatal age or 36 weeks post-conceptional age; group 2 consisted of eight babies who were oxygen dependent at 28 days postnatal age but not 36 weeks post-conceptional age; group 3 consisted of 11 babies who developed BPD (oxygen dependent at 36 weeks post-conceptional age); group 4 consisted of seven babies who died. Three babies died from progressive respiratory failure at 15,16 , and 22 days of age, and three died from acute respiratory failure within the first 24 hours of life. One died from necrotising enterocolitis having suffered minimal respiratory problems.

\section{Bronchoalveolar lavage}

BAL was conducted within the first 24 hours (where possible) and daily for the first seven days. Thereafter, it was conducted weekly for as long as the infant remained on the ventilator. It was performed at the time that suction/ lavage was clinically indicated and never within four hours of surfactant administration. The infant was supine with head turned to the left. Two separate aliquots of $1 \mathrm{ml} / \mathrm{kg}$ sterile normal saline were instilled into the lung through a 5FG DeeLee suction catheter (Pennine Ltd, Derby, UK) inserted down the endotracheal tube and wedged in a bronchus. BAL fluid was immediately aspirated under suction $\left(50 \mathrm{~cm} \mathrm{H}_{2} \mathrm{O}\right)$ into a bronchoscopy trap (Wallace UK, 3S Healthcare, London, UK) after each instilled aliquot. The two aliquots were pooled. Part of the sample was taken for bacterial culture, and the remainder was centrifuged at $1000 \mathrm{~g}$ for five minutes to remove mucous and cellular debris. The supernatant was stored at $-80^{\circ} \mathrm{C}$ before biochemical analysis.

\section{Blood sampling}

In those babies with an arterial line, about $400 \mu$ l blood was removed at the time of lavage. The sample was placed in a BD Microtainer (lithium heparin with serum separator) and centrifuged at $1000 \mathrm{~g}$ for five minutes to pellet the cells. The plasma was removed and stored at $-80^{\circ} \mathrm{C}$ before biochemical analyses.
Biochemical analyses

Initial processing of BAL fluid and plasma

A $200-250 \mu \mathrm{l}$ sample of BAL fluid supernatant was mixed with an equal volume of $0.2 \mathrm{M}$ perchloric acid to provide a final concentration of $0.1 \mathrm{M}$ perchloric acid. The sample was centrifuged at $10000 \mathrm{~g}$ for five minutes to pellet the protein. Then $50 \mu \mathrm{l}$ of the supernatant was removed and added to $450 \mu \mathrm{l}$ water for the measurement of glutathione. The remainder was used for the measurement of ascorbate and urate.

A $100 \mu \mathrm{l}$ sample of plasma was mixed with $100 \mu \mathrm{l} 1.0 \mathrm{M}$ perchloric acid, mixed well, and centrifuged at $10000 \mathrm{~g}$ for five minutes to pellet the protein. The supernatant was removed and diluted 1:5 with water to provide a final perchloric acid concentration of $0.1 \mathrm{M}$. A $100 \mu \mathrm{l}$ portion of the supernatant was added to $900 \mu \mathrm{l}$ water for the measurement of glutathione. The remainder was used to measure ascorbate and urate.

Urea was measured using neat BAL fluid supernatant or plasma. Malondialdehyde was measured using neat BAL supernatant.

Samples were stored for no longer than one month before analysis. Standards and spiked samples stored under the same conditions as the BAL and plasma samples were stable for at least this period. This is in agreement with previous studies. ${ }^{26} 27$

\section{Measurement of antioxidants}

Ascorbate and urate concentrations in the perchloric acid extracts of BAL fluid and plasma were measured by the HPLC-EC method of Mitton and Trevithick. ${ }^{28}$ The amount of ascorbate and urate in each sample was computed by comparison with standard curves prepared with each batch of samples. The limit of sensitivity of the technique was well below that required for this study. The lowest standards needed for both antioxidants was $100 \mathrm{nM}$.

Total glutathione concentrations in the perchloric acid extracts were measured by a previously developed method. ${ }^{29}$ The amount of glutathione present in samples was quantified against standard curves prepared with each batch of samples. As with the measurement of ascorbate and urate, the limit of sensitivity of the method was well below that needed for this study. The lowest standard used was $1 \mathrm{nM}$.

\section{Measurement of malondialdehyde}

Malondialdehyde was measured as authentic malondialdehyde not a thiobarbituric acid adduct (which is non-specific) by a modification of the method of Esterbauer et al. ${ }^{30}$ In our modification, an Aquasep column was used rather than an aminophase column. This provided more stable retention times. This required a modification of the mobile phase. The system consisted of a $150 \times 4.6 \mathrm{~mm}$ column of Aquasep C8 ( $3 \mu \mathrm{m}$ particle size; Hichrom) equipped with a guard column of the same material. In addition, because undiluted BAL supernatant was injected into the system, an in-line filter was included to protect the chromatographic system. The mobile phase was $0.03 \mathrm{M}$ Tris/HCl buffer, $\mathrm{pH}$ 6.5. The Rheodyne injection system incorporated a $20 \mu \mathrm{l}$ sample loop. Chromatography was conducted at room temperature at a flow rate of $1.0 \mathrm{ml} / \mathrm{min}$ using a Gynkotec P-580 solvent delivery system. Malondialdehyde was detected using a UVD $170 \mathrm{~S}$ detector (Gynkotec) at a wavelength of $267 \mathrm{~nm}$. The system was controlled using a Chromeleon chromatography control system (Dionex). The amount of malondialdehyde present in samples was quantified against standard curves prepared with each batch of samples. The limit of sensitivity was $10 \mathrm{nM}$. 


\section{Estimation of ELF volume}

There is still uncertainty about whether it is advisable to present data from BAL studies unadjusted or to express it as ELF concentrations. ${ }^{31}$ To permit comparison with other studies, we have expressed data as both ELF concentrations using the urea dilution technique ${ }^{32}$ to calculate the amount of ELF in each BAL sample, and also as unadjusted BAL.

\section{Measurement of urea}

Urea was measured in neat BAL fluid and plasma using a commercially available kit (Procedure No 535; Sigma Diagnostics, Sigma-Aldrich, Poole, Dorset, UK). For measuring urea in plasma, the kit was used according to the manufacturer's instructions. For measuring urea in BAL fluid, the technique was modified as follows: $100 \mu \mathrm{l}$ filtered BAL fluid $(0.2 \mu \mathrm{m})$ was added to $750 \mu \mathrm{l}$ blood urea nitrogen (BUN) acid reagent and $500 \mu \mathrm{l}$ BUN colour reagent. The mixture was heated at $100^{\circ} \mathrm{C}$ for 15 minutes and then cooled before reading at $535 \mathrm{~nm}$ in a Camspec spectrophotometer. The amount of urea present in the samples was quantified against standard curves prepared with each batch of samples.

The limit of sensitivity of the methods used was $10 \mu \mathrm{M}$ for $\mathrm{BAL}$, and the lowest standard used for plasma was $500 \mu \mathrm{M}$.

\section{Chemicals}

All chemicals used in this study were obtained from Fisher Scientific, Sigma, or Aldrich chemical companies.

\section{Statistical analysis}

All the ratio data were analysed using analysis of variance with Tukey's multiple comparison test, a two tailed $t$ test (where relevant), and by Pearson's correlation test. Nominal data were analysed by the Kruskall-Wallis test and, where significant differences were observed, the individual groups were compared by the Mann-Whitney U test.

Binary logistic regression analysis was carried out to determine which factors were predictive of the development of oxygen dependency at 36 weeks. Univariate analyses were conducted using Stat 100 (Biosoft) or SPSS. Multivariate analyses were conducted using SPSS. $\mathrm{p}<0.05$ was regarded as significant.

\section{RESULTS}

Table 1 shows the clinical and biochemical data for the four groups. There were no differences in sex, mode of delivery, and Apgar score between the four groups. The incidence of endotracheal infection and septicaemia was significantly higher in the babies who developed BPD than in those who did not $(p<0.02)$ and those who were oxygen dependent at 28 days $(\mathrm{p}<0.05)$ (Kruskall-Wallis and Mann-Whitney $U$ test). Ureaplasma infection was detected in similar proportions of the babies in the four groups. There was a significant increase in the volume and number of blood transfusions in babies who went on to develop BPD $(\mathrm{p}<0.05)$.

\section{ELF/BAL antioxidant concentrations}

As expected, the sickest babies required ventilation for a longer period and consequently provided the most BAL samples. The percentage recovery of BAL in this study was greater in the groups of babies with poorest clinical outcome.

There was no correlation between gestational age and the ELF/BAL concentration of any of the antioxidants.

The initial antioxidant concentrations (within the first 24 hours) expressed as both ELF or uncorrected BAL showed little difference in the concentrations of ascorbate and urate across the groups, but the concentration of glutathione was lowest in those babies who developed BPD. This reached significance when expressed as ELF concentrations ( $\mathrm{p}=$ 0.043 ). There was a significant correlation between the time to first surfactant administration and the initial ELF glutathione concentration $(r=0.47, \mathrm{p}<0.05)$.

\section{Plasma antioxidant concentrations}

Plasma glutathione was also significantly reduced in those babies who developed BPD $(p=0.022)$ and correlated significantly with time to first surfactant administration $(r=0.55, \mathrm{p}<0.01, \mathrm{n}=43)$. There was no correlation between plasma glutathione and BAL or ELF concentrations. In contrast, there was a positive correlation between plasma and BAL urate $(r=0.527, \mathrm{p}<0.001, \mathrm{n}=100)$, plasma and ELF urate $(r=0.406, \mathrm{p}<0.001, \mathrm{n}=99)$, plasma and BAL ascorbate $(r=0.419, \mathrm{p}<0.001, \mathrm{n}=100)$, and plasma and ELF ascorbate $(r=0.616, \mathrm{p}<0.001, \mathrm{n}=99)$. The concentration of glutathione in ELF was significantly greater than that in plasma, but the concentrations of ascorbate and urate were similar in plasma and ELF. There was a significant positive correlation between ascorbate and urate concentrations in both ELF $(r=0.63, \mathrm{p}<0.01)$ and BAL $(r=0.65$, $\mathrm{p}<0.01, \mathrm{n}=43)$.

\section{ELF/BAL malondialdehyde concentrations}

The mean malondialdehyde concentration of all BAL samples taken were significantly higher in babies who developed BPD than in those who did not $(\mathrm{p}<0.05)$.

\section{Changes in antioxidant/malondialdehyde concentrations with postnatal age}

Figure 1 shows changes in ELF concentrations of ascorbate, urate, and malondialdehyde during the first week of ventilation in those babies who were ventilated for seven days or more. Glutathione concentrations are not shown, as these changed little over the first week. The initial concentration of malondialdehyde was low and peaked at day 5. In contrast, both ascorbate and urate concentrations decreased over the first week. A similar trend is seen when the data are expressed as uncorrected BAL concentrations. In those babies still ventilated on day $14(\mathrm{n}=5)$, the urate and ascorbate concentrations remained low (mean (SEM) urate 77.69 (23.0) $\mu \mathrm{M}$; ascorbate $5.18(2.29) \mu \mathrm{M})$, and the malondialdehyde concentration remained high $(2.06(0.43) \mu \mathrm{M} ; \mathrm{n}=5)$. Plasma concentrations of ascorbate and urate also fell during the first five days of life, but glutathione concentrations remained stable (no baby had an arterial line in situ for more than five consecutive days). The data shown in fig 1 may predict an inverse relation between malondialdehyde and ascorbate and urate concentrations. However, this was not significant (Pearson's correlation test).

\section{Correlation of antioxidant/malondialdehyde concentrations with oxygen exposure and infection} There was no correlation between the degree of oxygen exposure (measured by mean fraction of inspired oxygen $\left(\mathrm{FIO}_{2}\right)$ in the six hours before taking each BAL sample as well as the peak $\mathrm{FIO}_{2}$ in the preceding 24 hours) and BAL/ELF antioxidant or malondialdehyde concentrations. There was a significant correlation between ELF/BAL malondialdehyde concentrations and the occurrence of endotracheal infection (ELF $r=0.38, \mathrm{p}<0.05$; BAL $r=0.37, \mathrm{p}<0.05, \mathrm{n}=35$ ).

\section{Binary logistic regression analysis}

It is clear that there are likely to be a number of covariances between the variables recorded. In an attempt to tease out which of these observations were most predictive of the development of BPD, the variables were subjected to binary logistic regression. The main predictors of the development of BPD were gestational age ( $\mathrm{z}$ value $3.07 ; \mathrm{p}<0.002$; odds ratio $0.30 ; 95 \%$ confidence interval (CI) 0.14 to 0.65 ), endotracheal infection ( $\mathrm{z}$ value $2.78 ; \mathrm{p}<0.005$; odds ratio $25.79 ; 95 \% \mathrm{CI}$ 
Table 1 Clinical and biochemical data according to outcome

\begin{tabular}{|c|c|c|c|c|}
\hline & Group $1(n=17)$ & Group $2(n=8)$ & Group $3(n=11)$ & Group $4(n=7)$ \\
\hline Gestational age (weeks) & $29(26-31)$ & $27(26-28)^{*}$ & $26(24-29)^{*}$ & $25(24-28)^{*}$ \\
\hline Birth weight (g) & $1306(845-2300)$ & $920(754-1200)^{*}$ & $773(535-1476)^{*}$ & $635(464-834)^{*}$ \\
\hline Peak $\mathrm{FlO}_{2}$ in first 24 hours & $44(21-97)$ & $42(22-69)$ & $43(21-66)$ & $62(32-100)$ \\
\hline Peak inspiratory pressure in first 24 hours & $18(14-30)$ & $17(12-20)$ & $18(14-24)$ & $21(18-26)$ \\
\hline First estimated $\mathrm{A} /$ a ratio & $0.31(0.13-0.51)$ & $0.28(0.19-0.35)$ & $0.40(0.18-0.76)$ & $0.18(0.1-0.27) \dagger$ \\
\hline No of blood transfusions received & $0.3(0-2)$ & $1.9(1-5)$ & $8.8(0-16)^{*}$ & $5.4(0-13)^{*}$ \\
\hline Total volume of blood received (ml) & $6.9(0-24)$ & $28.0(15-67)^{*}$ & $135.4(0-271)^{*}$ & $58.4(0-115)^{*}$ \\
\hline No of BAL samples taken & $1.7(1-4)$ & $3.3(1-5)$ & $4.9(2-9)$ & $4.0(1-7)$ \\
\hline Return of BAL fluid $(\%)$ & $36.6(21.7-65.5)$ & $43.9(24.0-60.6)$ & $56.0(28.5-74.0)$ & $73.4(63.0-99.0)$ \\
\hline \multicolumn{5}{|l|}{ Glutathione concentration $(\mu M)$} \\
\hline ELF & $10.76(2.97)$ & $4.19(2.16)$ & $1.89(0.62)^{*}$ & $2.96(0.96)$ \\
\hline BAL & $0.653(0.231)$ & $0.174(0.077)$ & $0.093(0.039)$ & $0.115(0.038)$ \\
\hline Plasma & $0.693(0.168)$ & $0.294(0.115)$ & $0.192(0.063)^{*}$ & $0.267(0.073)$ \\
\hline \multicolumn{5}{|l|}{ Ascorbate concentration $(\mu \mathrm{M})$} \\
\hline ELF & $27.57(9.04)$ & $31.72(12.01$ & $30.34(12.48)$ & $15.24(4.28)$ \\
\hline BAL & $1.32(0.26)$ & $1.10(0.46)$ & $1.54(0.51)$ & $0.94(0.36)$ \\
\hline Plasma & 71.05 (13.35) & $59.83(15.79)$ & $60.54(11.50)$ & $69.42(23.93)$ \\
\hline \multicolumn{5}{|l|}{ Urate concentration $(\mu \mathrm{M})$} \\
\hline ELF & $261.91(40.68)$ & $495.21(98.13)$ & $331.65(49.29)$ & $215.25(37.25)$ \\
\hline $\mathrm{BAL}$ & $17.33(2.11)$ & $19.53(3.07)$ & $14.20(2.61)$ & $10.35(1.73)$ \\
\hline Plasma & $540.46(44.12)$ & $534.35(98.54)$ & $381.59(47.52)^{*}$ & $439.23(65.26)$ \\
\hline \multicolumn{5}{|l|}{ Malondialdehyde concentration } \\
\hline ELF $(\mu M)$ & $0.345(0.095)(n=16)$ & $1.150(0.577)(n=3)$ & $1.300(0.313)(n=10)^{*}$ & $0.912(0.364)(n=6)$ \\
\hline BAL $(n M)$ & $18.43(5.02)(n=16)$ & $18.18(1.57)(n=3)$ & $52.76(13.22)(n=10)^{*}$ & $36.01(13.87)(n=6)$ \\
\hline \multicolumn{5}{|c|}{$\begin{array}{l}\text { Clinical data are expressed as mean (range). Initial (within } 24 \text { hours of birth) antioxidant concentration and mean malondialdehyde concentration are expressec } \\
\text { as mean (SEM). } \\
\text { *Significantly }(p<0.05) \text { different from group } 1 . \\
\text { †Significantly }(\mathrm{p}<0.05) \text { different from group } 3 \text {. } \\
\text { Group 1, Babies who survived without } \mathrm{O}_{2} \text { requirement; group 2, } \mathrm{O}_{2} \text { dependent at } 28 \text { days postnatal age but not at } 36 \text { weeks post-conceptional age; group } 3, \mathrm{O} \\
\text { dependent at } 36 \text { weeks post-conceptional age (BPD); group 4, babies who died. }\end{array}$} \\
\hline
\end{tabular}

2.62 to 254.98 ), and septicaemia ( $\mathrm{z}$ value $2.44 ; \mathrm{p}<0.01$; odds ratio 17.64; $95 \%$ CI 1.76 to 177.12$)$. Gestational age essentially soaked up the available power to predict the occurrence of BPD. However, if this variable were omitted, then the inclusion of endotracheal infection or septicaemia provided as powerful a predictive model as any other. There was evidence of some limited predictive power for the glutathione and malondialdehyde variables but this failed to reach significance. These effects were unchanged whether or not the three babies who died from progressive respiratory failure at 15, 16, and 22 days of age were included in the BPD group.

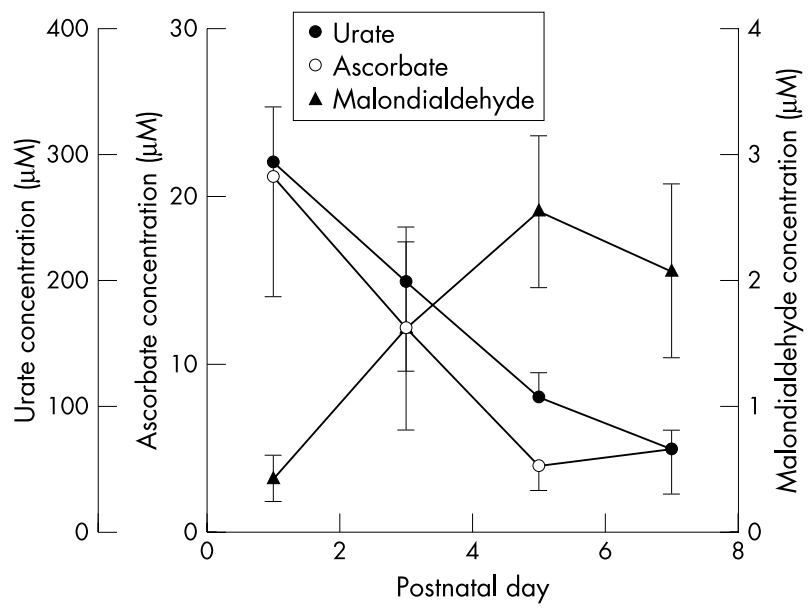

Figure 1 Concentrations of urate, ascorbate, and malondialdehyde in the first week of life in the total number of babies $(n=8)$ who were ventilated for more than a week. Malondialdehyde concentration was measured in only five of these. Data are consequently expressed as mean (SEM) for eight babies for urate and ascorbate, and mean (SEM) for five babies for malondialdehyde.

\section{DISCUSSION}

The purpose of this study was to examine the relation between pulmonary extracellular antioxidants, oxidative damage, and clinical outcome. In particular, the study looked at antioxidant concentrations in the early days of life. Antioxidant concentrations supplied to the baby at birth may be clinically important for allowing it to cope with the rigours of extrauterine life. Extracellular antioxidant and malondialdehyde concentrations were sampled by BAL. The higher percentage recovery of BAL in babies with poor clinical outcome is probably due to a greater amount of residual fluid in the bronchoalveolar spaces of these babies because of greater vascular and epithelial permeability. ${ }^{34}$ This transvascular fluid flux should be accompanied by low molecular mass solutes such as urea. Accordingly, the calculation of ELF volumes using urea dilution should provide reasonably accurate figures for the concentration of antioxidants and malondialdehyde in the fluid lining the bronchoalveolar spaces.

The initial concentrations of ELF urate and ascorbate were similar to those seen in adults (ascorbate 40 (18) $\mu \mathrm{M}$; urate $207(167) \mu \mathrm{M}),{ }^{35}$ but glutathione concentrations were at least one order of magnitude lower (adult value 109 (64) $\mu \mathrm{M}$ )..$^{35}$ The finding that low initial glutathione concentrations are associated with the development of BPD is consistent with previous observations, ${ }^{18}{ }^{19}$ although a recent study reported no such difference. ${ }^{28}$ Babies with higher initial glutathione concentrations did not require surfactant treatment until later in their clinical course, suggesting that these babies had less severe lung disease. Animal studies have shown the importance of glutathione as a pulmonary antioxidant, ${ }^{16} 1736$ and there is evidence of a deficiency of glutathione in adult pulmonary conditions in which oxidative damage plays a role. ${ }^{37}$ Although plasma glutathione concentrations were also low in babies who developed BPD, the lack of correlation between BAL/ELF and plasma concentrations (in contrast with urate and ascorbate) suggests that pulmonary concentrations are regulated independently of plasma concentrations. The much higher concentration of ELF glutathione 
compared with plasma concentrations suggests that it is actively secreted into the alveolar spaces.

The higher mean concentration of malondialdehyde in babies who develop BPD indicates a higher level of oxidative damage during their time on the ventilator. Previous studies using other markers of oxidative damage have produced similar results. ${ }^{8} 9839$ However, despite this evidence, binary logistic regression analysis showed that the ability of these variables (glutathione and malondialdehyde) to predict the development of BPD was weak. In a multifactorial condition such as BPD, it is perhaps naïve to expect clear predictive outcomes from such data. It is nevertheless likely that deficiency of glutathione does contribute to BPD by compromising pulmonary antioxidant capacity. ${ }^{18} 19$

Although the concentration of ELF and BAL malondialdehyde is higher in those babies with poor clinical outcome, very few babies are born with high malondialdehyde concentrations. In those babies in which sufficient sequential samples were taken to allow measurements to be made over the first week, the concentration of malondialdehyde rose over the first few days of life. During this time, glutathione concentrations changed little, but urate and ascorbate concentrations fell, as has been noted in previous studies. ${ }^{12} 28$

The two likely causes of oxidative damage in these babies are free radicals generated as a result of hyperoxia and respiratory burst activity of invading inflammatory cells. ${ }^{40} \mathrm{We}$ found no correlation between the inspired oxygen concentrations and BAL/ELF antioxidant or malondialdehyde concentrations. There was, however, a strong correlation between endotracheal infection/septicaemia and ELF/BAL malondialdehyde concentrations, suggesting that the inflammatory response to infection is more likely than hyperoxia to lead to oxidative damage. The pattern of malondialdehyde production, with higher concentrations occurring at around day 5 rather than at birth would be consistent with this. This is further supported by the results of the logistic regression analysis, which indicated that endotracheal infection in combination with septicaemia and gestational age was a powerful predictor of the development of BPD.

\section{ACKNOWLEDGEMENTS}

We gratefully thank the staff and parents at the Neonatal Unit, The Royal Devon \& Exeter Hospital for their assistance with this research. This work was supported by grants from the NHS Executive South West Region and the London Law Trust.

\section{Authors' affiliations}

K J Collard, Department of Medical Sciences, St Loye's School of Health Studies, Millbrook House, Exeter EX2 6ES, UK

S Godeck, J E Holley, M W Quinn, Department of Child Health, School of Postgraduate Medicine, University of Exeter, Church Lane, Heavitree, Exeter EX2 5SQ

\section{REFERENCES}

1 Singer L, Yamashita T, Lilian L, et al. A longitudinal study of developmental outcome of infants with bronchopulmonary dysplasia and very low birth weight. Pediatrics 1997; 100:987-93.

2 Farel AM, Hooper SR, Teplin SW, et al. Very low birth weight infants at seven years: an assessment of health and neurodevelopmental risk conveyed by chronic lung disease. J Learn Disabil 1998:31:118-26.

3 Kornhauser MS, Baumbart S, Desai SA, et al. Adverse neurodevelopmental outcome after extracorporeal membrane oxygenation among neonates with bronchopulmonary dysplasia. J Pediatr 1998;132:307-1 1.

4 Abman SH, Groothius JR. Pathophysiology and treatment of bronchopulmonary dysplasia. Pediatr Clin N Am 1994;41:347-415.

5 Fardy CH, Silverman M. Antioxidants in neonatal lung disease. Arch Dis Child Fetal Neonatal Ed 1995;73:F112-17.

6 Saugstadt OD. Bronchopulmonary dysplasia and oxidative stress: are we closer to an understanding of the pathogenesis of BPD? Acta Paediatr 1997;86:1277-82.
7 MacNee W. Oxidants/antioxidants and COPD. Chest 2000;117:303S-17S.

8 Inder TE, Graham P, Sanderson K, et al. Lipid peroxidation as a measure of oxygen free radical damage in the very low birth weight infant. Arch Dis Child Fetal Neonatal Ed 1994;70:F107-11.

9 Varsila E, Pesonen E, Anderson S. Early protein oxidation in the neonatal lung is related to development of chronic lung disease. Acta Paediatr 1995; 84: 1296-9.

10 VanKlaveren RJ, Demedts $M$, Nemery B. Cellular glutathione turnover in vitro, with emphasis on type II pneumocytes. Eur Respir J 1997; 10:1392-40.

11 Becker BF, Reinholz N, Liepert B, et al. Role of uric acid as an endogenous radical scavenger. Chest 1991;100:176S-81S

12 Schrod L, Neuhaus T, Speer CP, et al. Possible role of uric acid as an antioxidant in premature infants. Biol Neonate 1997;72:102-11.

13 Maritz GS. Ascorbic acid: protection of lung tissue against damage. Subcellular Biochem 1996;25:265-91.

14 Cantin AM, Begin R. Glutathione and inflammatory disorders of the lung. Lung $1991 ; 169: 123-38$

15 Meister A. On the antioxidant effects of ascorbic acid and glutathione. Biochem Pharmacol 1992;44:1905-15.

16 Martensson J, Jain A, Stole E, et al. Inhibition of glutathione synthesis in the newborn rat: a model for endogenously produced oxidative stress. Proc Natl Acad Sci USA 1991;88:9360-4.

17 Chessex P, Lavoie JC, Laborie S, et al. Survival of guinea pig pups in hyperoxia is improved by enhanced nutritional substrate availability for glutathione production. Pediatr Res 1999;46:305-10.

18 Grigg J, Barber A, Silverman M. Bronchoalveolar lavage fluid glutathione in intubated premature infants. Arch Dis Child 1997;69:49-51.

19 Reise JA, Taylor GW, Fardy CH, et al. Glutathione and neonatal lung disease. Clin Chim Acta 1997;265:113-19.

20 Sevanian A, Davies KJA, Hochstein P. Serum urate as an antioxidant for ascorbic acid. Am J Clin Nutr 1991;54:1129S-34S.

21 Halliwell B, Gutteridge JMC. The antioxidants of human extracellular fluids. Arch Biochem Biophys 1990;280:1-8

22 Cini M, Fariello RG, Bianchetti A, et al. Studies on lipid peroxidation in the rat brain. Neurochem Res 1994; 19:283-8.

23 Lazzarino G, Tavazzi B, Di Pierro D, et al. The relevance of malondialdehyde as a biochemical index of lipid peroxidation of post ischaemic tissues in the rat and human being. Biol Trace Elem Res 1995;47:165-70.

24 Schlenzig JS, Bervoets K, Van Leowenich V, et al. Urinary malondialdehyde concentration in preterm neonates: is there a relationship to disease entities of neonatal intensive care? Acta Paediatr 1993;82:202-5.

25 Godeck S, Collard K, Quinn M. Informed parental consent in neonatal research: time consuming but feasible? Proceedings of the British Association of Perinatal Medicine, September Meeting 2000

26 Vyas JR, Currie A, Dunster C, et al. Ascorbic acid concentration in airways lining fluid from infants who develop chronic lung disease of prematurity. Eur J Pediatr 2001;160:177-84.

27 Young IS, Trimble ER. Measurement of malondialdehyde in plasma by high performance liquid chromatography with fluorimetric detection. Ann Clin Biochem 1991;28:504-8.

28 Mitton KP, Trevithick JR. High performance liquid chromatographyelectrochemical detection of antioxidants in vertibrate lens: glutathione, tocopherol and ascorbate. Methods Enzymol 1994;223:523-39.

29 Nanda D, Tolputt J, Collard KJ. Changes in brain glutathione levels during postnatal development in the rat. Dev Brain Res 1996;94:238-41.

30 Esterbaver $\mathbf{H}$, Lang J, Zadraver S, et al. Detection of malondialdehyde by high performance liquid chromatography. Methods Enzymol 1984;105:319-28.

31 ERS Task Force. Bronchoalveolar lavage in children. Eur Respir J 2000;15:217-31.

32 Rennard SI, Basset G, Lecossier D, et al. Estimation of volume of epithelial lining fluid recovered by lavage using urea as a marker of dilution. J Appl Physiol 1986;60:532-8

33 Jobe $\mathbf{A}$, Jacobs $H$, Ikegami $M$, et al. Lung protein leaks in ventilated lambs: effect of geststional age. J Appl Physiol 1985;58:1246-51.

34 Nama V, Kozlowski JK, Hamvas A. Measurement of protein flux with positron emission tomography in neonates. Arch Dis Child Fetal Neonatal Ed 1999;80:F26-9.

35 Van Der Vliet A, O'Neill CA, Cross CE, et al. Determination of low molecular mass antioxidant concentrations in human respiratory tract lining fluids. Am J Physiol Lung Cell Mol Physiol 1999;20:L289-96.

36 Jain A, Martensson J, Mehta T, et al. Ascorbic acid prevents oxidative stress in glutathione-deficient mice: effects on lung type II cell lamellar bodies, lung surfactant and skeletal muscle. Proc Nat Acad Sci USA 1992;89:5093-7.

37 Tager M, Piecyk A, Kohnlein T, et al. Evidence of defective thiol status in alveolar macrophages from COPD patients and smokers. Free Radic Biol Med 2000;29:1 160-5.

38 Pitkanen OM, Hallman M, Anderson SM. Correlation of free oxygen radicalinduced lipid peroxidation with outcome in very low birth weight infants. J Pediatr 1990; 116:760-4

39 Schock BC, Sweet DG, Halliday HL, et al. Oxidative stress in lavage fluid of preterm infants at risk of chronic lung disease. Am J Physiol Lung Cell Mol Physiol 2002;281:L1386-91

40 Contreras M, Hariharan N, Lewandoski JR, et al. Bronchoalveolar oxyradical inflammatory elements herald bronchopulmonary dysplasia. Crit Care Med 1996;24:29-37. 